\title{
Spatio Temporal Parameters and Symmetry Index in Transtibial Amputees Wearing Prosthetic Feet with and without Adaptive Ankles
}

\author{
M. Riveras ${ }^{1,2}$, E. Ravera ${ }^{1,2}$, A. F. Shaheen ${ }^{3,4}$, D. Ewins ${ }^{4}$, P. Catalfamo Formento ${ }^{1,2,4}$ \\ ${ }^{1}$ IBB, CONICET-UNER, Oro Verde, Argentina \\ ${ }^{2}$ LIMH, FIUNER, Oro Verde, Argentina \\ ${ }^{3}$ University of Brunel, London, UK \\ ${ }^{4}$ University of Surrey, Guildford, UK \\ Email: mriveras@ingenieria.uner.edu.ar; pcatalfamo@ingenieria.uner.edu.ar ;
}

\begin{abstract}
This study measured spatio temporal parameters (STP) and their symmetry index (SI) in order to evaluate the differential effect on the gait pattern of individuals with unilateral transtibial amputations when using two different prostheses. Twelve individuals with transtibial amputations walked on level ground using an Energy Storage and Return (ESAR) prosthesis with fixed ankle and a prosthetic foot with adaptive ankle (PFAA). The STP were measured in the prosthetic and sound limbs and the symmetry index for each parameter was calculated afterwards. The results showed no statistically significant differences between the prostheses for the STP measured, and this was the case both for the prosthetic and sound limbs. Similarly, the SI did not reflect statistically significant differences when the different prostheses were used. Thus, the results suggest that the STP studied and their SI may not reflect differences when evaluating ESAR versus PFAA prostheses in the conditions proposed in this study.
\end{abstract}

Keywords - Adaptative ankle prosthesis, Energy storage and return ankle prosthesis, Transtibial amputee.

\section{INTRODUCTION}

Gait analysis can be defined as the systematic study of human walking, performed by a combination of observations by experienced clinicians and instrumented measurements [1]. In the clinical treatment of patients with conditions that affect their ability to walk, it could be used for example, to make a detailed assessment of the causes of the walking pattern and to plan an optimal treatment [1], [2].

In the area of amputee rehabilitation, health professionals have suggested the use of gait analysis for gaining basal information about the patient, assessing progress in the rehabilitation process, selecting prosthetic components and for demonstrating effectiveness of intervention, among others [3], [4]. An area of interest in amputee rehabilitation is the selection of the optimal prosthesis for a particular patient and also the evaluation of advantages and limitations of the different type of prostheses [5].

Prosthetic devices aim at enabling individuals with amputations to successfully return to functional and recreational activities. Many of these devices, particularly those for individuals with transtibial amputations, do not fully mimic the function of unimpaired joints and have been associated with gait deviations and functional limitations, such as decreased walking speed [6] and increased interlimb asymmetries in spatiotemporal parameters and kinetics of the joints [7].

Different types of prosthetic feet are prescribed for transtibial amputees. Of those, "Energy storage and return" (ESAR) feet are capable of mechanically storing elastic energy generated during the early stance and midstance and then returning it in late stance and preswing to provide some of the mechanical energy normally provided by the ankle plantarflexors [8], [9]. One of these devices is the Esprit ${ }^{\mathrm{TM}}$ foot (Chas. A. Blatchford and Sons Ltd., Basingstoke, UK). Another type of prosthesis is the Prosthetic Foot with Adaptive Ankles (PFAA). It allows for an increased range of motion of the ankle to improve walking performance. One of these devices is the Echelon ${ }^{\mathrm{TM}}$ foot (Chas. A. Blatchford and Sons Ltd., Basingstoke, UK) which allows for a hydraulically controlled stance-phase articulation. When set-up correctly, an Echelon foot provides $6^{\circ}$ plantarflexion and $3^{\circ}$ dorsiflexion relative to its neutral (standing) position [10].

A variety of gait analysis tools have been proposed for characterizing gait in amputees, including questionnaires for evaluating the degree of adaptation to the prosthethic [11][13], tests such as the AMPPRO [14] and POGS [15], spatio temporal parameters (STP), kinematic and kinetic parameters [3], [4], [16]. Using different tools, studies have been carried out comparing the performances between ESAR and PFAA hydraulic prostheses in transtibial unilateral amputees. Some studies have investigated toe clearance during swing phase as an indicator of the likelihood of falling [17], others studied the transition of the center of pressure [18] and its fluctuations [10], or the foot braking effect during stance [19]. Also, satisfaction levels have been requested from amputees, when comparing the prosthesis for many facets of prosthetic use during the activities of daily living [20].

Of the gait analysis tools proposed for gait analysis, STP have been reported as the most commonly used biomechanical parameter for the evaluation of lower limb amputees [16], [21]. The comparison of STP between the prosthetic and sound limb, measured by the symmetry index 
also provides useful information. For example, often the stance phase for the sound limb of lower limb amputees is slightly longer than on the prosthetic side, contributing to a more asymmetrical gait [22], [23]. This is partly caused by subjects relying more on their sound leg to compensate for some of the deficiencies associated with prostheses. The increased loading period may explain the development of complications in the remaining limb [24], hence gait asymmetry is one of the concerns in unilateral lower limb amputees when endeavoring to minimize excessive load on the sound limb [25], [26].

STP and their SI are appealing for routine clinical use. The main reasons for this is that the equipment required to collect the data is often portable, relatively low cost, easy to use and do not require long measurement times. However, not many studies have evaluated STP for comparison between ESAR and PFAA. Moore [18] showed a reduction in asymmetry of stance phase duration when using hydraulic PFAA prostheses when compared with ESAR. However, no other data from STP parameters, such as Swing Time or Double Support, or symmetry were reported. Also, it has been reported that when comparing different prostheses, some studies found statistically significant differences in the STP while others did not [21]. Many causes may influence this variability, including the type of prosthesis used, the number and physical conditions of the participants, and the level of amputation, among others.

The collection and use of STP therefore appears to be a relatively straightforward option for clinical assessment. However, their ability to measure effects on gait pattern when using prosthetic feet with and without adaptive ankles has not been fully evaluated. This paper aims at measuring STP and their symmetry in order to evaluate differential effects on the gait pattern of two different prostheses (ESAR and PFAA) in physically active transtibial amputees. The results would add information that could help decide whether STP and their symmetry indices may be considered as an outcome measure in the clinical environment for selection of the most appropriated prosthetic foot.

\section{METHODOLOGY}

\section{A. Participants}

Twelve physically active (9 males, 3 females), unilateral transtibial amputees, (mean (SD) age 41.5 (15.5) years, mass $72.0(13.2) \mathrm{kg}$, height $1.75(0.09) \mathrm{m})$ were involved in this study. They had used a prosthetic foot for at least four months (12.2 (12.9) years, range 0.33-47 years). Each participant gave written informed consent prior to their involvement. Local ethics committee approval was obtained for the protocol.

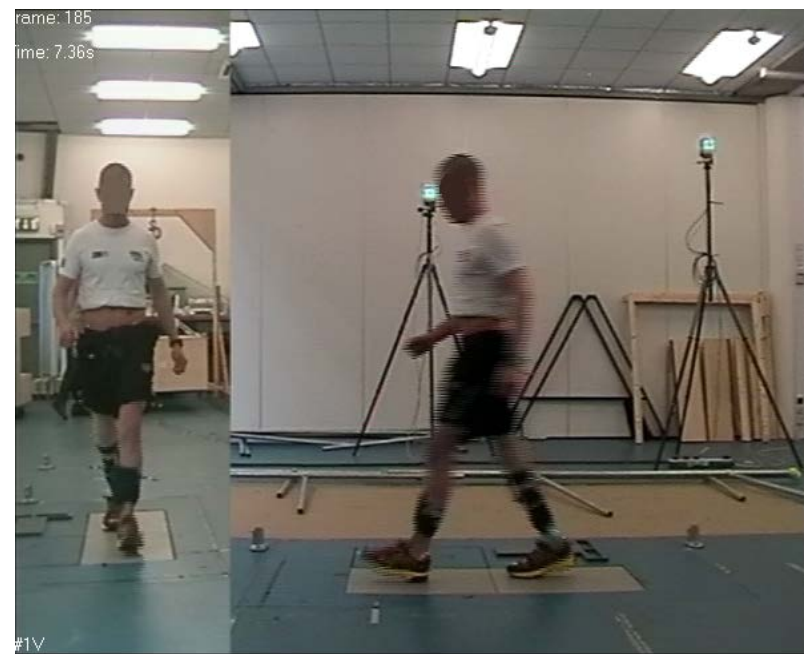

Fig. 1. Measurement setup during walking of the participants with transtibial amputation.

B. Protocol and prosthetic intervention

Subjects walked on a 10 meter pathway using two different prostheses: an ESAR and a PFAA (Fig. 1). The prostheses were the Esprit and Echelon models, respectively, selected from the Endolite family (Chas. A. Blatchford and Sons Ltd., Basingstoke, UK).

The first prosthesis used in the experiment was the one not normally used by the participant. So if they habitually used an ESAR foot, the PFAA foot was fitted first and vice versa. After fitting the non-habitual prosthesis, a minimum time of one hour was given for the subject to become familiar with it.

The fitting of the prosthesis was performed by an experienced prosthetist, who was careful to ensure the best possible alignment and adjustment for each foot. The socket, suspension and alignment of the shank pylon were unchanged across foot types. The settings which control the rates of articulation within the hydraulic feet (damping) were adjusted by the prosthetist until deemed to provide optimal function at a self-selected, comfortable walking speed.

\section{Data acquisition and processing}

Participants walked in a straight line along the pathway at their self-selected comfortable walking speed as recommended in the literature [27]. At least 6 trials were performed by each participant with each prosthesis. Kinematic and kinetic data were recorded at $200 \mathrm{~Hz}$ and 400 $\mathrm{Hz}$ respectively using an eleven camera motion capture system (ProReflex, Qualisys, Göteborg, Sweden) and two force platforms (AMTI, MA, USA). During data collection, participants wore their own comfortable shoes. 

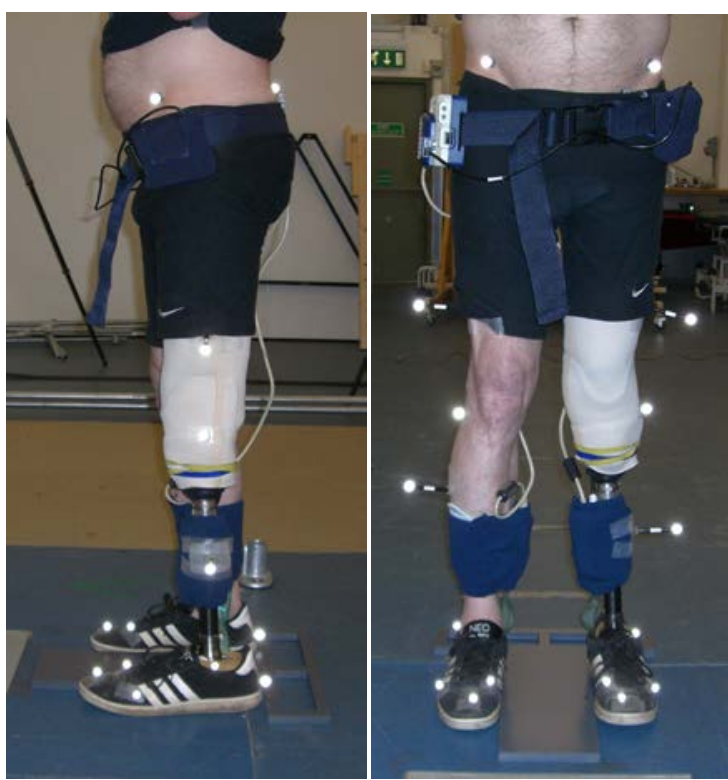

Fig. 2. Helen Hayes marker set used in the study.

Using the Helen Hayes marker set (Fig. 2), 19 spherical retroreflective markers (all $14 \mathrm{~mm}$ diameter except markers placed onto the feet which were $9.5 \mathrm{~mm}$ diameter) were bilaterally attached to the lower limbs of each participant, (or equivalent locations on the prosthesis). Two additional markers were placed on the toe and at equivalent locations on the prosthetic side.

Initial contact (IC) and foot off (FO) were defined using kinematic data, as proposed by Zeni et al [28]. Walking speed, cycle time, step length, double support, stance time and swing time were calculated using the software Visual3D v6TM (Version 6.01.08, C-Motion, Germantown, MD, USA). The parameters were then normalized in order to avoid bias in the results. Step length was normalized to subject height and the temporal parameters were normalized to cycle time [29], using MatLab (R2016a, The MathWorks, Inc., Natick, Massachusetts, United States).

Symmetry Index (SI) for all the STP was defined as shown in Eq. (1) [25]

$$
\begin{aligned}
& \text { Eq. (1): } \\
& \qquad S I=\frac{\min (P R, P L)}{\max (P R, P L)} * 100
\end{aligned}
$$

Where SI stands for the symmetry index and PR, PL stand for the values of the gait parameter measured for the right and left limb, respectively [25].

The mean value for each participant was calculated for all parameters and each prosthesis using at least 25 strides per subject. Later, the median value for all twelve participants was calculated.

\section{Statistical analyses}

Data from STP and SI were statistically analyzed with the non-parametric Wilcoxon test for paired samples and an alpha level set at 0.05, using SPSS (23.0.0.0, IBM, Armonk New York U.S.A.). The effect size (W) [30], was calculated. Values of $\mathrm{W}$ will be considered in case of $\mathrm{p}$ value lower than 0.05. Median, first quartile (q1) and third quartile (q3) were used as statistical descriptors.

\section{RESULTS}

\section{A. Spatio Temporal parameters}

Table I shows the results of the STP for the sound and prosthetic limbs, for both prostheses. The table shows the median values and (q1 q3) for each parameter, for each type of prosthesis studied. Also the statistical p value and the effect size (W) of the comparison between prosthesis are reported.

The median values of the parameters did not show statistically significant differences between the prostheses for any of the parameters studied.

\section{B. Symmetry Index}

Table II shows the median values of symmetry indices and (q1 q3) for the parameters for each type of prosthesis studied. Also reported are the statistical p value obtained and the effect size $(\mathrm{W})$ of the comparison between prosthesis.

The median values of the SI did not show statistically significant differences between the prostheses for any of the studied parameters.

\section{DISCUSSION}

The spatiotemporal parameters found for each group in this study for the prosthetic and sound side are in agreement with results previously reported in the literature for transtibial amputees [31]-[33].

In terms of the comparison between prosthesis, walking speed increased when using a PFAA with respect to ESAR, which is consistent with previous findings [10], [19]. Gard [21] suggested that walking speed may be the most appropriate way of assessing prosthetic performance since it might be expected that self-selected walking speed would increase if a prosthetic configuration helps produce a more efficient pattern of walking. Given that the PFAA allow for an increased range of motion of the ankle intending to improve walking performance, it was expected to find an increase in self-selected walking speed when using this prosthesis. 
TABLE I

MEDiAN VALUE, FIRST QUARTILE AND THE THIRD QUARTILE (q1 q3) FOR STEP LENGTH (SL), EXPRESSED AS \% OF BODY HEIGHT (BH), DOUBLE SUPPORT (DS), STANCE TIME (ST) AND SWING TIME (SWT), EXPRESSED IN \% OF GAIT CYCLE, AND SPEED [m/s]. N=12.

\begin{tabular}{|c|c|c|c|c|c|c|c|c|}
\hline \multicolumn{5}{|c|}{ Prosthetic limb } & \multicolumn{4}{|c|}{ Sound limb } \\
\hline Parameter & ESAR & PFAA & $\mathrm{p}$ & $\mathrm{W}$ & ESAR & PFAA & $\mathrm{p}$ & $\mathrm{W}$ \\
\hline SL & 41.7 & 42.0 & 0.136 & 0.41 & 38.1 & 39.1 & 0.433 & 0.22 \\
\hline (q1 q3) & (37.3 44.8) & (37.9 45.8) & & & (36.7 41.4) & (36.5 42.0) & & \\
\hline DS & 17.0 & 18.0 & 0.05 & 0.54 & 16.2 & 15.1 & 0.084 & 0.48 \\
\hline (q1 q3) & (16.0 17.7) & (16.2 19.0) & & & (15.2 18.2) & (13.5 17.6) & & \\
\hline ST & 65.7 & 65.8 & 0.814 & 0.07 & 67.4 & 67.2 & 0.388 & 0.24 \\
\hline (q1 q3) & (64.9 66.9) & (65.5 66.8) & & & (66.5 68.7) & (65.7 68.0) & & \\
\hline SwT & 34.3 & 34.0 & 0.695 & 0.11 & 32.7 & 33.0 & 0.272 & 0.3 \\
\hline (q1 q3) & (33.3 34.7) & (33.6 34.4) & & & (31.6 33.4) & (32.4 34.0) & & \\
\hline Speed & 1.284 & 1.332 & 0.158 & 0.39 & & & & \\
\hline (q1 q3) & (1.162 1.323) & (1.209 1.424) & & & & & & \\
\hline
\end{tabular}

However, this improvement was not reflected in the STP. In fact, the differences between prosthesis in temporal parameters (Stance Time, Swing Time and Double Support) and in Step Length were small (around 1\% of Gait Cycle and $1 \%$ of Height, respectively). Consistently with these small differences, the statistical tests did not show significant differences for any of the parameters studied when comparing the prosthesis.

The median values of SI were larger than $83 \%$ for all prostheses and all parameters. These results are in agreement to those reported in the literature for rehabilitated transtibial amputees while walking on level ground which show symmetry values greater than $80 \%$ [25], [34], [35]. This is slightly lower than the SI found in healthy people, for whom the SI between dominant and non-dominant foot was found to be greater than 94\% [35], [36]. However, once again the differences in SI between PFAA and ESAR were relatively small and the statistical tests did not show statistically significant differences for SI between the prostheses.

Some considerations should be taking into account.

The number of participant on this study was similar to other studies in the literature reporting differences in gait conditions for transtibial amputees [9], [34], [37]-[40]. Although more statistical power could be gained from a larger sample size, the sample size used in this study should be enough as indicative of a tendency in the results. In term of the gender of the participants there are no suggestions in the literature that gait patterns may present differences between male and female. This is the reason for including both in the present study.

The inclusion criteria of the study selected physically active participants, as a first approached to evaluated differences between prosthesis. However, the levels of activity of the volunteers ranged from people who were able to walk independently in their activities of daily life (K2) to people participating regularly in highly demanding sports activities, such as triathlons (K4). This wide range of mobility may have affected the variability of the data.

Future work should, if possible, plan the analysis on a $\mathrm{K}$ level basis. This is not always practicable, as it was not for this study, given the relatively low number of participants and the time and effort required to recruit more volunteers. 
MEDIAN VALUES, FIRST QUARTILE AND THE THIRD QUARTILE (q1 q3) OF S.I. FOR STEP LENGTH (SL), DOUBLE SUPPORT (DS), STANCE TIME (ST), SWING TIME (SWT) AND CYCLE TIME (CT).

\begin{tabular}{lcccc}
\hline \multicolumn{1}{c}{ Parameter } & ESAR & PFAA & p & W \\
\hline SL & 90.3 & 90.6 & 0.875 & 0.04 \\
\multicolumn{1}{c}{ q1 q3 } & $(86.992 .7)$ & $(86.894 .8)$ & & \\
DS & 88.5 & 83.8 & 0.117 & 0.44 \\
\multicolumn{1}{c}{ q1 q3 } & $(82.093 .9)$ & $(70.990 .4)$ & & \\
ST & 96.3 & 97.1 & 0.48 & 0.2 \\
q1 q3 & $(95.397 .2)$ & $(95.297 .9)$ & & \\
SwT & 93.6 & 95.5 & 0.158 & 0.39 \\
q1 q3 & $(92.595 .5)$ & $(92.996 .8)$ & & \\
CT & 98.2 & 98.2 & 0.754 & 0.09 \\
q1 q3 & $(97.598 .7)$ & $(97.898 .8)$ & & \\
\hline
\end{tabular}

It has also been reported that experienced prosthetic users are able to adapt to prosthetic modifications relatively fast without showing deterioration in their gait, but rather displaying the same gait pattern as before [21]. Clearly there should be appropriate gait retraining in order to take full advantage of new prosthetic features that could allow the amputee to develop trust, security and confidence with the new technology. Not all participants of this study had received gait training for both prosthetic feet, rather some of them had used only one of the two prosthetic feet evaluated. Therefore, it is possible that evaluating the effect on gait without an appropriate retraining may not reflect real changes in the walking pattern. Participants of this study had an hour for familiarization with the non-habitual prosthesis but some of them had not received rehabilitation treatment with that particular prosthesis.

Finally, reports in the literature found that subjects often express clear preference for one prosthesis over another, even when quantitative gait analysis does not show statistically significant differences, suggesting that very subtle changes in gait may be detected by the user and be perceived as significant [21], [41]. Focusing the evaluation in areas of perceived significance may be a more appropriate approach for the evaluation of prosthesis, for example by expanding test environments to measure activities where the amputee perceived improvement such as high-velocity motions, such as running, or different terrains.

Taking into account all these considerations, together with the fact that the parameters measured in this study are those commonly used for characterizing amputee gait, but they have not reflected differences when evaluating prostheses in the conditions of this study future work should focus on evaluating the parameters in different conditions. First, the evaluation should be performed in settings of perceived significance, such as incline or stairs walking or high velocity motions.

\section{CONCLUSION}

This paper assessed spatio temporal parameters and their symmetry index in order to evaluate the differential effects on the gait pattern of physically active transtibial amputees when using two different prostheses (ESAR and PFAA).

None of the measured parameters and symmetry indices showed statistically significant differences. From these results, it is possibly to conclude that although the parameters measured in this study are those commonly used for evaluating and characterizing amputee gait, they may not reflect differences when evaluating prostheses in the conditions proposed in this study.

The results of this research suggest that for clinical evaluating differences between prosthesis using STP and their SI it is possible that the condition of the environment should be different (including ramps or stairs, and different walking speed conditions).

\section{ACKNOWLEDGMENT}

The authors would like to thank the participants who volunteered to participate in this study.

\section{REFERENCES}

[1] Michael W. Whittle, GAIT ANALYSIS AN INTRODUCTION, Fourth. Heidi Harrison, 2007.

[2] R. W. Baker, Measuring Walking: A Handbook of Clinical Gait Analysis, First. Mac Keith Press, 2013.

[3] M. J. M. J. Cole et al., "BACPAR Toolbox of outcome measures. Version 2,” vol. 2015, no. Accessed on February 2015. 2014.

[4] A. Esquenazi, "Gait analysis in lower-limb amputation and prosthetic rehabilitation,” Phys. Med. Rehabil. Clin. N. Am., vol. 25, no. 1, pp. 153-167, 2014.

[5] D. Rusaw and N. Ramstrand, "Motion-analysis studies of transtibial prosthesis users: a systematic review.," Prosthet. Orthot. Int., vol. 35, no. 1, pp. 8-19, 2011.

[6] Y. Hermodson, C. Ekdahl, B. M. Persson, and G. Roxendal, "Gait in male trans-tibia1 amputees : a comparative study with healthy subjects in relation to walking speed,” pp. 68-77, 1994.

[7] G. R. B. Hurley, R. Mckenney, M. Robinson, M. Zadravec, and M. R. Pierrynowski, "The role of the contralateral limb in belowknee amputee gait,” Prosthet. Orthot. Int., vol. 14, no. 1, pp. 3342, 1990.

[8] B. J. Hafner, "Clinical Prescription and Use of Prosthetic Foot and Ankle Mechanisms: A Review of the Literature," JPO J. Prosthetics Orthot., vol. 17, no. Supplement, pp. S5-S11, 2005.

[9] R. J. Zmitrewicz, R. R. Neptune, J. G. Walden, W. E. Rogers, and G. W. Bosker, "The Effect of Foot and Ankle Prosthetic Components on Braking and Propulsive Impulses During Transtibial Amputee Gait,” Arch. Phys. Med. Rehabil., vol. 87, no. 10, pp. 1334-1339, 2006.

[10] A. R. De Asha, L. Johnson, R. Munjal, J. Kulkarni, and J. G. Buckley, "Attenuation of centre-of-pressure trajectory fluctuations under the prosthetic foot when using an articulating hydraulic ankle attachment compared to fixed attachment," Clin. Biomech., vol. 28, no. 2, pp. 218-224, 2013.

[11] P. Gallagher and M. MacLachlan, "Trinity Amputation and Prosthesis Experience Scales - Revised,” vol. 89, no. 2010, pp. 506-513, 2005. 
[12] F. A. de Laat, G. M. Rommers, J. H. Geertzen, and L. D. Roorda, "Construct Validity and Test-Retest Reliability of the Walking Questionnaire in People With a Lower Limb Amputation," Arch. Phys. Med. Rehabil., vol. 93, no. 6, pp. 983-989, 2012.

[13] R. S. Hanspal, K. Fisher, and R. Nieveen, "Prosthetic socket fit comfort score.,” Disabil. Rehabil., vol. 25, no. 22, pp. 12781280, 2003.

[14] R. S. Gailey et al., "The Amputee Mobility Predictor: An instrument to assess determinants of the lower-limb amputee's ability to ambulate," Arch. Phys. Med. Rehabil., vol. 83, no. 5, pp. 613-627, May 2002.

[15] S. J. Hillman et al., "Repeatability of a new observational gait score for unilateral lower limb amputees," Gait Posture, vol. 32, no. 1, pp. 39-45, 2010.

[16] Y. Sagawa, K. Turcot, S. Armand, A. Thevenon, N. Vuillerme, and E. Watelain, "Biomechanics and physiological parameters during gait in lower-limb amputees: a systematic review.," Gait $\{\&\}$ posture, vol. 33, no. 4, pp. 511-526, 2011.

[17] L. Johnson, A. R. De Asha, R. Munjal, J. Kulkarni, and J. G. Buckley, "Toe clearance when walking in people with unilateral transtibial amputation: Effects of passive hydraulic ankle," vol. 51, no. 3, pp. 429-438, 2014.

[18] R. Moore, "Effect on Stance Phase Timing Asymmetry in Individuals with Amputation Using Hydraulic Ankle Units," $J$. Prosthetics Orthot., vol. 28, no. 1, pp. 44-48, 2016.

[19] A. R. De Asha, R. Munjal, J. Kulkarni, and J. G. Buckley, "Impact on the biomechanics of overground gait of using an 'Echelon' hydraulic ankle-foot device in unilateral trans-tibial and trans-femoral amputees.," Clin. Biomech. (Bristol, Avon), vol. 29, no. 7, pp. 728-734, Jun. 2014.

[20] I. Sedki and R. Moore, "Patient evaluation of the Echelon foot using the Seattle Prosthesis Evaluation Questionnaire," Prosthet. Orthot. Int., vol. 37, no. 3, pp. 250-254, 2013.

[21] S. a. Gard, "Use of Quantitative Gait Analysis for the Evaluation of Prosthetic Walking Performance," JPO J. Prosthetics Orthot., vol. 18, pp. P93-P104, 2006.

[22] D. J. Sanderson and P. E. Martin, "Lower extremity kinematic and kinetic adaptations in unilateral below-knee amputees during walking," Gait Posture, vol. 6, no. 2, pp. 126-136, 1997.

[23] E. Isakov, H. Burger, J. Krajnik, M. Gregoric, and C. Marincek, "Influence of speed on gait parameters and on symmetry in transtibial amputees.," Prosthet. Orthot. Int., vol. 20, no. 3, pp. 153158, 1996

[24] Y. Sagawa, K. Turcot, S. Armand, A. Thevenon, N. Vuillerme, and E. Watelain, "Biomechanics and physiological parameters during gait in lower-limb amputees: A systematic review," Gait Posture, vol. 33, no. 4, pp. 511-526, 2011.

[25] G. N. S. Marinakis, "Interlimb symmetry of traumatic unilateral transtibial amputees wearing two different prosthetic feet in the early rehabilitation stage,” J. Rehabil. Res. Dev., vol. 41, no. 4, p. 581, 2004.

[26] L. Nolan, "The functional demands on the intact limb during walking for active trans-femoral and trans-tibial amputees," Prosthet. Orthot. Int., vol. 24, pp. 117-125, 2000.

[27] A. R. De Asha, R. Munjal, J. Kulkarni, and J. G. Buckley, "Walking speed related joint kinetic alterations in trans-tibial amputees: impact of hydraulic 'ankle' damping,” J. Neuroeng. Rehabil., vol. 10, pp. 107-122, 2013.

[28] J. A. Zeni Jr, J. G. Richards, and J. S. Higginson, “Two Simple Methods for Determining Gait Events During Treadmill and Overground Walking Using Kinematic Data," Gait Posture, vol. 27, no. 4, p. 710, 2008.

[29] J. Cámara Tobalina, "Gait analysis: phases and spatio-temporal variables,” Entramado, vol. 7, no. 1, pp. 160-173, 2011.

[30] M. Tomczak and E. Tomczak, "The need to report effect size estimates revisited. An overview of some recommended measures of effect size,” Trends Sport Sci., vol. 1, no. 21, pp. 19-25, 2014. I. Kova, V. Medved, and L. Ostoji, "Spatial, temporal and kinematic characteristics of traumatic transtibial amputees' gait.," Coll. Antropol., vol. 34, no. Supplement 1, pp. 205-213, 2010.
[32] S. J. Olney, "Kinematic and Kinetic Variations of Below-Knee Amputee Gait Take Online Quiz for PCEs,” vol. 14, no. 1, pp. 113, 2014

[33] H. Sadeghi, P. Allard, and M. Duhaime, "Muscle power compensatory mechanisms in below-knee amputee gait," Am. $J$. Phys. Med. Rehabil., vol. 80, no. 1, pp. 25-32, 2001.

[34] M. J. Highsmith, B. W. Schulz, S. Hart-Hughes, G. A. Latlief, and S. L. Phillips, "Differences in the spatiotemporal parameters of transtibial and transfemoral amputee gait," J. Prosthetics Orthot., vol. 22, no. 1, pp. 26-30, 2010.

[35] L. Nolan, A. Wit, K. Dudziñski, A. Lees, M. Lake, and M. Wychowañski, "Adjustments in gait symmetry with walking speed in trans-femoral and trans-tibial amputees," Gait Posture, vol. 17, no. 2, pp. 142-151, Apr. 2003.

[36] M. Blazkiewicz, I. Wiszomirska, and A. Wit, "Comparison of four methods of calculating the symmetry of spatial-temporal parameters of gait,” Acta Bioeng. Biomech., vol. 16, no. 1, pp. 29-35, 2014.

[37] D. H. Gates, J. B. Dingwell, S. J. Scott, E. H. Sinitski, and J. M. Wilken, "Gait characteristics of individuals with transtibial amputations walking on a destabilizing rock surface," Gait Posture, vol. 36, no. 1, pp. 33-39, 2012.

[38] N. J. Rosenblatt, A. Bauer, D. Rotter, and M. D. Grabiner, "Active dorsiflexing prostheses may reduce trip-related fall risk in people with transtibial amputation,” J. Rehabil. Res. Dev., vol. 51, no. 8, pp. 1229-1242, 2014.

[39] V. Agrawal, R. S. Gailey, I. A. Gaunaurd, C. O’Toole, A. Finnieston, and R. Tolchin, "Comparison of four different categories of prosthetic feet during ramp ambulation in unilateral transtibial amputees,” Prosthet. Orthot. Int., vol. 39, no. 5, pp. 380-389, 2015.

[40] A. Eshraghi, N. A. Abu Osman, M. Karimi, H. Gholizadeh, E. Soodmand, and W. A. B. Wan Abas, "Gait biomechanics of individuals with transtibial amputation: Effect of suspension system," PLoS One, vol. 9, no. 5, pp. 1-12, 2014.

[41] B. J. Hafner, J. E. Sanders, J. M. Czerniecki, and J. Fergason, "Energy storage and return prostheses: Does patient perception correlate with biomechanical analysis?," Clin. Biomech., vol. 17, no. 5, pp. 325-344, 2002. 